Molecules 2001, 6, 540-550

molecules

ISSN 1420-3049

http://www.mdpi.org

\title{
Alkylation of Zwitterionic Thiooxalic Acid Derivatives
}

\section{Klaus Martens ${ }^{1}$, Anke Scheunemann ${ }^{1}$, Kerstin Drexler ${ }^{1}$, Heinz Dehne ${ }^{1, *}$, Helmut Reinke ${ }^{1}$ and Manfred Michalik ${ }^{2}$}

${ }^{1}$ Fachbereich Chemie der Universität Rostock, Buchbinderstr. 9, D-18055 Rostock, Germany. Tel.: +49-381-498-1767, Fax: +49-381 4981763.

${ }^{2}$ Institut für Organische Katalyseforschung an der Universität Rostock, Buchbinderstr. 5-6, D-18055

Rostock, Germany.

*Author to whom correspondence should be addressed; E-mail: heinz.dehne@ chemie.uni-rostock.de.

Received: 12 March 2001 / Accepted: 10 May 2001 / Published: 31 May 2001

\begin{abstract}
The new S-alkyl thiooxal-1-hydrazono-2-amidrazonium halides 2-4 were synthesized by reaction of the corresponding zwitterionic thiooxalic acid derivatives $\mathbf{1}$ with alkyl halides in methanol. The structures of compounds $\mathbf{4 b}$ and $\mathbf{4 d}$ were proven by X-ray structural analysis. Both compounds form an interesting intermolecular network of hydrogen bonds in the solid state.
\end{abstract}

Keywords: Alkylation, thiooxalic acid derivatives, halides, X-ray analysis.

\section{Introduction}

Generally thiocarboxamides and their anions react with alkyl halides in kinetically controlled reactions at the S-atom. With alkylating agents which first form stable carbenium ions, direct $\mathrm{N}$ alkylation was observed. However, in the alkylation reactions of alkane and arene thiocarboxylic acid hydrazides only S-alkylation has been found [1]. 1,4-Dihydrotetrazines were formed by selfcondensation and elimination of alkanethiol from the thiocarboxylic acid S-alkyl ester hydrazonides, which were obtained by reaction of $\mathrm{N}$-unsubstituted thiohydrazides with alkyl halides in alkaline medium [2]. The S-alkylation products of $\mathrm{N}^{2}$-mono- and $\mathrm{N}^{2}, \mathrm{~N}^{2}$-disubstituted thiocarboxylic acid 
hydrazides were thermally more stable [1]. Some years ago we synthesized the new zwitterionic thiooxalic acid 2-amide-1-hydrazide-2-hydrazone (1a) [3] and the methyl (1b) and phenyl derivative (1c), respectively [4]. These compounds are multifunctional building blocks especially for heterocycles $[5,6]$. Here we describe reactions of the substances 1a-c with several alkyl halides and discuss some structural data of the new alkylation products.

\section{Results and Discussion}

The zwitterionic thiooxalic acid derivatives 1a-c reacted in methanol with several alkyl halides to give the yellow S-alkyl-thiooxal-1-hydrazono-2-amidrazonium halides 2-4 in good yields (Scheme 1).

Scheme 1. Synthesis of the halides 2-4<smiles>[R]NN[C@H](N)[C@@H](S)N[R]</smiles>

1

a: $\mathrm{R}^{1}=\mathrm{H}$

b: $\mathrm{R}^{1}=\mathrm{CH}_{3}$

c: $\mathrm{R}^{1}=\mathrm{C}_{6} \mathrm{H}_{5}$

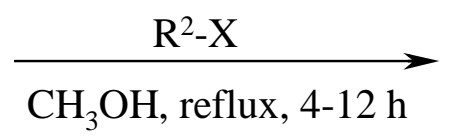

$\mathrm{CH}_{3} \mathrm{OH}$, reflux, 4-12 h

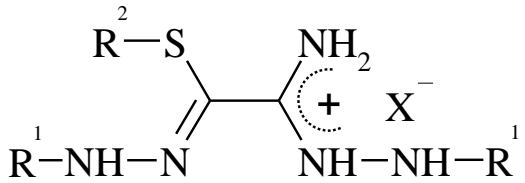

2-4

$\mathrm{X}=\mathrm{Br}, \mathrm{I}$

While the educts 1a and $\mathbf{1 b}$ only needed $4 \mathrm{~h}$ under reflux to react (Experimental, Method A) the more stable phenyl derivative 1c had to be heated for $12 \mathrm{~h}$ (Experimental, Method B). The reaction was performed in the absence of a strong base like potassium hydroxide because compounds $\mathbf{2 - 4}$ are unstable under these conditions. The best yields of the products were obtained with alkyl bromides and iodides (Table 1) but not with the corresponding chlorides.

Table 1. Analytical data of the halides 2-4

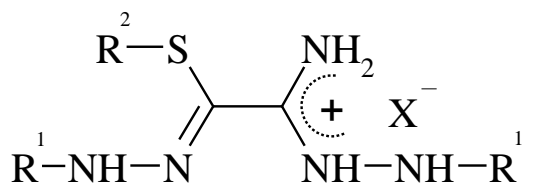

\begin{tabular}{|c|c|c|c|c|cccc|}
\hline \multirow{2}{*}{ Compd. } & Formula & $\mathbf{R}^{\mathbf{1}}$ & \multirow{2}{*}{ X } & M.p. ${ }^{\circ} \mathbf{C}$ & \multicolumn{4}{|c|}{ Calculated/Found } \\
& (M.w.) & $\mathbf{R}^{\mathbf{2}}$ & & Yield \% & \% C & \% H & \% N & \% S \\
\hline \multirow{2}{*}{$\mathbf{2 a}$} & $\mathrm{C}_{3} \mathrm{H}_{10} \mathrm{IN}{ }_{5} \mathrm{~S}$ & $\mathrm{H}$ & \multirow{2}{*}{$\mathrm{I}$} & $119-120$ & 13.09 & 3.66 & 25.45 & 11.65 \\
& $(275.11)$ & $\mathrm{CH}_{3}$ & & 59 & 13.34 & 3.65 & 25.41 & 11.65 \\
\hline \multirow{2}{*}{$\mathbf{2 b}$} & $\mathrm{C}_{9} \mathrm{H}_{13} \mathrm{BrN}_{6} \mathrm{O}_{2} \mathrm{~S}$ & $\mathrm{H}$ & \multirow{2}{*}{$\mathrm{Br}$} & $92-94$ & 30.95 & 3.75 & 24.06 & 9.18 \\
& $(349.20)$ & $4-\mathrm{NO}_{2} \mathrm{C}_{6} \mathrm{H}_{4} \mathrm{CH}_{2}$ & & 72 & 30.74 & 3.73 & 23.51 & 9.13 \\
\hline
\end{tabular}


Table 1. Continued

\begin{tabular}{|c|c|c|c|c|c|c|c|c|}
\hline 3 & $\begin{array}{c}\mathrm{C}_{11} \mathrm{H}_{17} \mathrm{BrN}_{6} \mathrm{O}_{2} \mathrm{~S} \\
(377.26)\end{array}$ & $\begin{array}{c}\mathrm{CH}_{3} \\
4-\mathrm{NO}_{2} \mathrm{C}_{6} \mathrm{H}_{4} \mathrm{CH}_{2}\end{array}$ & $\mathrm{Br}$ & $\begin{array}{c}87-90 \\
92 \\
\end{array}$ & $\begin{array}{l}35.02 \\
35.10 \\
\end{array}$ & $\begin{array}{l}4.54 \\
4.62\end{array}$ & $\begin{array}{l}22.28 \\
22.19\end{array}$ & $\begin{array}{l}8.50 \\
8.38 \\
\end{array}$ \\
\hline $4 a$ & $\begin{array}{c}\mathrm{C}_{15} \mathrm{H}_{18} \mathrm{IN}_{5} \mathrm{~S} \\
(427.30)\end{array}$ & $\begin{array}{l}\mathrm{C}_{6} \mathrm{H}_{5} \\
\mathrm{CH}_{3}\end{array}$ & I & $\begin{array}{c}196-200 \\
62\end{array}$ & $\begin{array}{l}42.16 \\
41.99\end{array}$ & $\begin{array}{l}4.25 \\
4.35 \\
\end{array}$ & $\begin{array}{l}16.39 \\
16.35\end{array}$ & $\begin{array}{l}7.50 \\
7.41\end{array}$ \\
\hline $4 b$ & $\begin{array}{c}\mathrm{C}_{16} \mathrm{H}_{20} \mathrm{IN}_{5} \mathrm{~S} \\
(441.33) \\
\end{array}$ & $\begin{array}{l}\mathrm{C}_{6} \mathrm{H}_{5} \\
\mathrm{C}_{2} \mathrm{H}_{5}\end{array}$ & I & $\begin{array}{c}195-200 \\
65 \\
\end{array}$ & $\begin{array}{l}43.54 \\
43.44 \\
\end{array}$ & $\begin{array}{l}4.57 \\
4.51 \\
\end{array}$ & $\begin{array}{l}15.87 \\
15.99 \\
\end{array}$ & $\begin{array}{l}7.26 \\
7.17 \\
\end{array}$ \\
\hline $4 c$ & $\begin{array}{c}\mathrm{C}_{24} \mathrm{H}_{36} \mathrm{BrN}_{5} \mathrm{~S} \\
(506.54)\end{array}$ & $\begin{array}{c}\mathrm{C}_{6} \mathrm{H}_{5} \\
\mathrm{CH}_{3}\left(\mathrm{CH}_{2}\right)_{9}\end{array}$ & $\mathrm{Br}$ & $\begin{array}{c}75-80 \\
78 \\
\end{array}$ & $\begin{array}{l}56.91 \\
56.76 \\
\end{array}$ & $\begin{array}{l}7.16 \\
7.14\end{array}$ & $\begin{array}{l}13.83 \\
13.30\end{array}$ & $\begin{array}{l}6.33 \\
6.15 \\
\end{array}$ \\
\hline $4 d$ & $\begin{array}{c}\mathrm{C}_{21} \mathrm{H}_{22} \mathrm{BrN}_{5} \mathrm{~S} \\
(456.41)\end{array}$ & $\begin{array}{c}\mathrm{C}_{6} \mathrm{H}_{5} \\
\mathrm{C}_{6} \mathrm{H}_{5} \mathrm{CH}_{2}\end{array}$ & $\mathrm{Br}$ & $\begin{array}{c}205-209 \\
40\end{array}$ & $\begin{array}{l}55.26 \\
55.30 \\
\end{array}$ & $\begin{array}{l}4.86 \\
4.96 \\
\end{array}$ & $\begin{array}{l}15.34 \\
15.34 \\
\end{array}$ & $\begin{array}{l}7.02 \\
7.03 \\
\end{array}$ \\
\hline $4 e$ & $\begin{array}{c}\mathrm{C}_{21} \mathrm{H}_{21} \mathrm{BrN}_{6} \mathrm{O}_{2} \mathrm{~S} \\
(501.40)\end{array}$ & $\begin{array}{c}\mathrm{C}_{6} \mathrm{H}_{5} \\
4-\mathrm{NO}_{2} \mathrm{C}_{6} \mathrm{H}_{4} \mathrm{CH}_{2}\end{array}$ & $\mathrm{Br}$ & $\begin{array}{c}140-145 \\
67\end{array}$ & $\begin{array}{l}50.31 \\
49.42\end{array}$ & $\begin{array}{l}4.22 \\
4.54\end{array}$ & $\begin{array}{l}16.76 \\
16.50\end{array}$ & $\begin{array}{l}6.39 \\
6.34\end{array}$ \\
\hline
\end{tabular}

The structures given for 2-4 are consistent with the spectroscopic data. Thus, in the ${ }^{1} \mathrm{H},{ }^{1} \mathrm{H}$ COSY spectrum of $4 \mathbf{a}$ a correlation between the $\mathrm{C}_{6} \mathrm{H}_{5} \mathrm{~N} \underline{\mathrm{HNH}}$ and $\mathrm{C}_{6} \mathrm{H}_{5} \mathrm{NHN} \underline{\mathrm{H}}$ protons was observed. In the HMBC spectrum of $\mathbf{4 a}$ correlations were found between $\mathrm{C}-1$ ' and the $\mathrm{C}_{6} \mathrm{H}_{5} \mathrm{~N}$ HNH proton as well as $\mathrm{S}-\mathrm{C}=\mathrm{N}$ and the $\mathrm{CH}_{3}$ protons, respectively (see Experimental). Thus, a distinction between the NH protons and the phenyl group signals could be made.

Figure 1. Arrangement of molecules of $\mathbf{4 b}$ in the crystal lattice

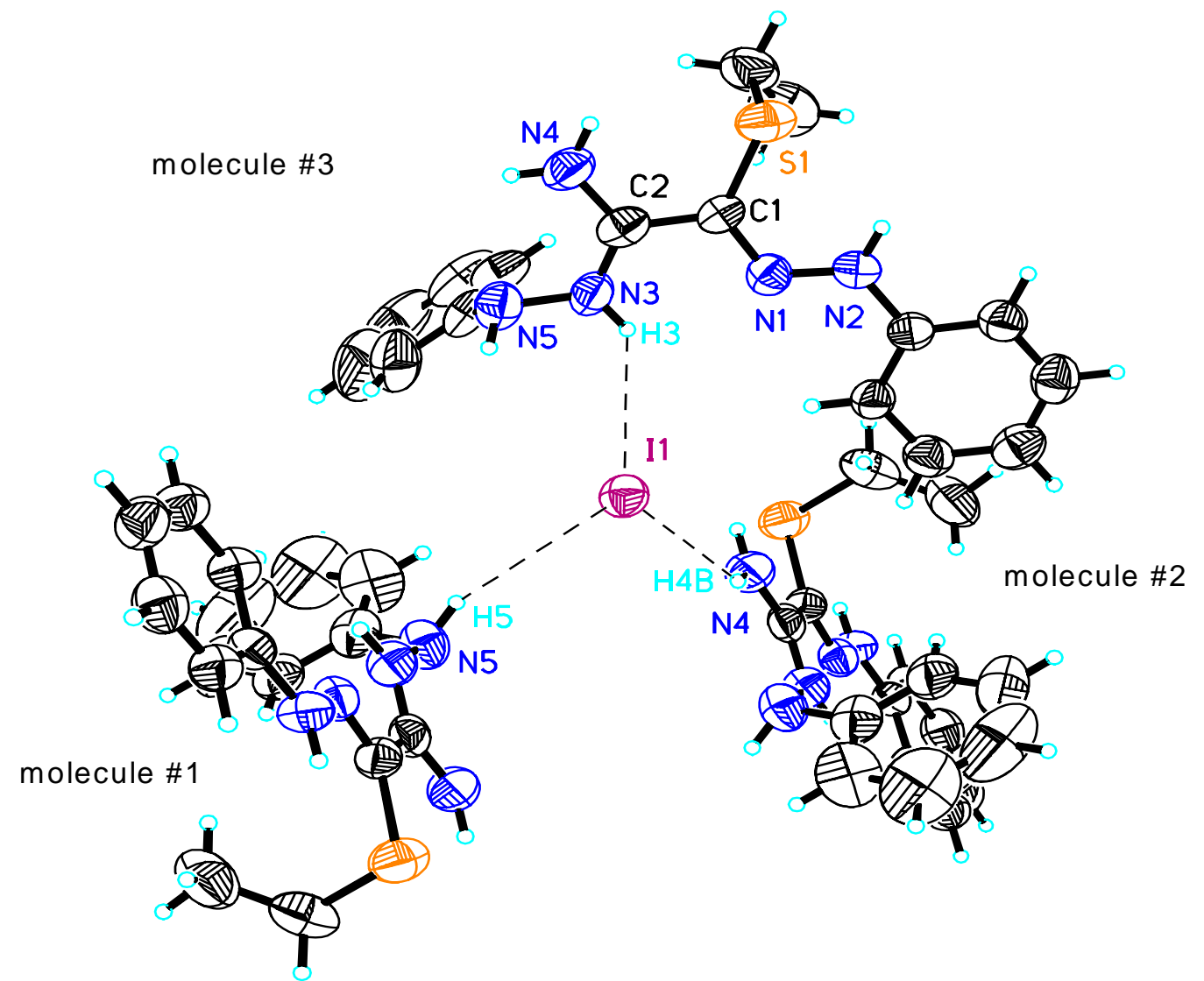


$\mathrm{X}$-ray structure investigations of the educt $\mathbf{1 c}$ and of the alkylation products $\mathbf{4 b}$ and $\mathbf{4 d}$ (see Figures 1 and 2, Table 2) demonstrate that the original conformation in the crystal of 1c [4] is rearranged in these alkylation products. Both compounds form an interesting intermolecular network of hydrogen bonds in the solid state. While the dominating motif in $\mathbf{4 b}$ consists of three organic molecules around one iodide with $\mathrm{N}(3) \mathrm{H}(3), \mathrm{N}(4) \mathrm{H}(4 \mathrm{~B}), \mathrm{N}(5) \mathrm{H}(5)$ as donor groups (see Figure 1, Table 3), the bromide in $\mathbf{4 d}$ is surrounded by four molecules (see Figure 2, Table 4).

Figure 2. Arrangement of molecules of $\mathbf{4 d}$ in the crystal lattice

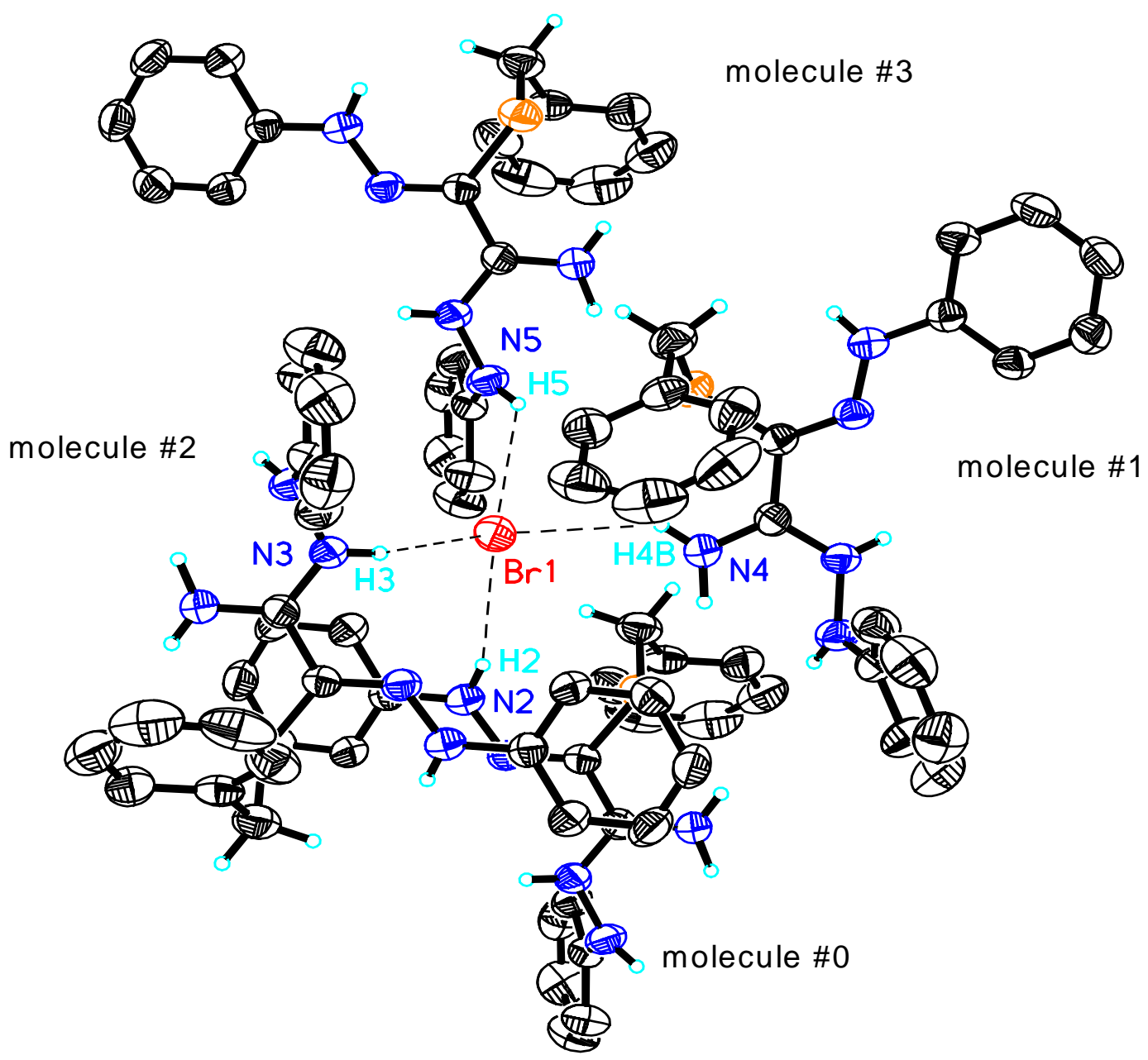

Each molecule provides another NH function so that all four NH-groups participate in the twodimensional network. A similar structure was already investigated earlier: the corresponding compound S-(methoxycarbonylmethyl)-thiooxal-1-(2-phenylhydrazono)-2-(2-phenylamidrazonium) bromide resulted from the reaction of $1 \mathbf{c}$ with methyl bromo acetate [5]. 
Table 2. Crystal structure data.

\begin{tabular}{|c|c|c|}
\hline Compound & $4 b$ & $4 d$ \\
\hline Empirical formula & $\mathrm{C}_{16} \mathrm{H}_{20} \mathrm{IN}_{5} \mathrm{~S}$ & $\mathrm{C}_{21} \mathrm{H}_{22} \mathrm{BrN}_{5} \mathrm{~S}$ \\
\hline Formula weight & 441.33 & 456.41 \\
\hline Crystal system & \multicolumn{2}{|c|}{ 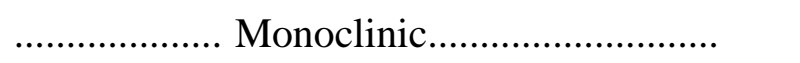 } \\
\hline Space group & $\mathrm{C} 2 / \mathrm{c}$ & $\mathrm{P} 2{ }_{1} / \mathrm{n}$ \\
\hline Unit cell dimensions $[\AA]$ & $a=22.963(5)$ & $13.391(3)$ \\
\hline & $\mathrm{b}=13.236(3)$ & $10.928(3)$ \\
\hline & $c=16.537(3)$ & $15.504(3)$ \\
\hline & $\beta=132.44(3)^{\circ}$ & $\beta=111.03(2)^{\circ}$ \\
\hline Volume $\left[\AA^{3}\right]$ & $3709.3(13)$ & 2117.7(9) \\
\hline$\rho$ (calculated) $\left[\mathrm{g} \mathrm{cm}^{-3}\right]$ & 1.581 & 1.432 \\
\hline $\mathrm{Z}$ & 8 & 4 \\
\hline $\mathrm{F}(000)$ & 1760 & 936 \\
\hline$\mu(\mathrm{Mo}-\mathrm{K} \alpha)\left[\mathrm{mm}^{-1}\right]$ & 1.845 & 2.056 \\
\hline Crystal size $[\mathrm{mm}]$ & $0.52 \times 0.44 \times 0.24$ & $0.52 \times 0.39 \times 0.30$ \\
\hline $2 \Theta$ range & $3.9 / 44$ & $4.68 / 45.98$ \\
\hline hkl range & $-24 / 1,-1 / 13,-13 / 17$ & $-1 / 14,-12 / 1,-17 / 16$ \\
\hline Measured refl. & 2750 & 3747 \\
\hline Unique refl. & 2271 & 2910 \\
\hline Observed refl. & 1948 & 2231 \\
\hline Completeness to $\Theta=22.00^{\circ}$ & $99.9 \%$ & $99.2 \%$ \\
\hline Data / restraints / parameters & $2271 / 0 / 228$ & $2910 / 0 / 257$ \\
\hline Min. transm. & 0.59421 & 0.12686 \\
\hline Max. transm. & 0.83244 & 0.16969 \\
\hline R1 for observed refl. & 0.0350 & 0.0433 \\
\hline R1 for all refl. & 0.0417 & 0.0643 \\
\hline wR2 for all refl. & 0.0930 & 0.1097 \\
\hline GoF on $\mathrm{F}^{2}$ & 1.022 & 1.009 \\
\hline$\rho(\max / \min )\left[\mathrm{e} . \AA^{-3}\right]$ & $0.374 /-0.442$ & $0.319 /-0.426$ \\
\hline
\end{tabular}

Table 3. Selected bond lengths [in $\AA$ ] , bond angles [in ${ }^{\circ}$ ] and torsion angles [in ${ }^{\circ}$ ] for compound $\mathbf{4 b}$

\begin{tabular}{llllll}
\hline $\mathrm{S}(1)-\mathrm{C}(1)$ & $1.763(5)$ & $\mathrm{N}(4)-\mathrm{C}(2)$ & $1.315(7)$ & $\mathrm{H}(2) \ldots \mathrm{S}(1)$ & 2.56 \\
$\mathrm{~N}(1)-\mathrm{C}(1)$ & $1.302(6)$ & $\mathrm{C}(1)-\mathrm{C}(2)$ & $1.462(7)$ & $\mathrm{N}(3)-\mathrm{H}(3) \ldots \mathrm{N}(1)$ & $2.680(6)$ \\
$\mathrm{N}(1)-\mathrm{N}(2)$ & $1.313(6)$ & $\mathrm{N}(4)-\mathrm{H}(4 \mathrm{~A}) \ldots \mathrm{S}(1)$ & $3.019(7)$ & $\mathrm{H}(3) \ldots \mathrm{N}(1)$ & 2.37 \\
$\mathrm{~N}(3)-\mathrm{C}(2)$ & $1.311(7)$ & $\mathrm{H}(4 \mathrm{~A}) \ldots \mathrm{S}(1)$ & 2.59 & $\mathrm{~N}(3)-\mathrm{C}(2)-\mathrm{N}(4)$ & $120.5(6)$ \\
$\mathrm{N}(3)-\mathrm{N}(5)$ & $1.408(7)$ & $\mathrm{N}(2)-\mathrm{H}(2) \ldots \mathrm{S}(1)$ & $2.959(5)$ & $\mathrm{N}(3)-\mathrm{C}(2)-\mathrm{C}(1)$ & $120.5(5)$ \\
\hline
\end{tabular}


Table 3. Continued

\begin{tabular}{llllll}
\hline $\mathrm{N}(4)-\mathrm{C}(2)-\mathrm{C}(1)$ & $119.0(6)$ & $\mathrm{N}(4)-\mathrm{H}(4 \mathrm{~B}) \ldots \mathrm{I}(1) \# 2$ & $3.539(6)$ & $\mathrm{C}(2)-\mathrm{C}(1)-\mathrm{S}(1)$ & $120.6(4)$ \\
$\mathrm{N}(2)-\mathrm{N}(1)-\mathrm{C}(1)-\mathrm{C}(2)$ & $-177.8(4)$ & $\mathrm{H}(4 \mathrm{~B}) \ldots \mathrm{I}(1) \# 2$ & 2.96 & $\mathrm{~N}(5)-\mathrm{N}(3)-\mathrm{C}(2)-\mathrm{C}(1)$ & $178.0(5)$ \\
$\mathrm{N}(2)-\mathrm{N}(1)-\mathrm{C}(1)-\mathrm{S}(1)$ & $1.7(6)$ & $\mathrm{N}(3)-\mathrm{H}(3) \ldots \mathrm{I}(1) \# 3$ & $3.518(5)$ & $\mathrm{N}(1)-\mathrm{C}(1)-\mathrm{C}(2)-\mathrm{N}(3)$ & $-3.1(7)$ \\
$\mathrm{N}(5)-\mathrm{N}(3)-\mathrm{C}(2)-\mathrm{N}(4)$ & $-3.7(8)$ & $\mathrm{H}(3) \ldots \mathrm{I}(1) \# 3$ & 2.83 & $\mathrm{~S}(1)-\mathrm{C}(1)-\mathrm{C}(2)-\mathrm{N}(3)$ & $177.4(4)$ \\
$\mathrm{N}(4)-\mathrm{H}(4 \mathrm{~B}) \ldots \mathrm{N}(5)$ & $2.678(9)$ & $\mathrm{C}(1)-\mathrm{N}(1)-\mathrm{N}(2)$ & $119.5(4)$ & $\mathrm{N}(1)-\mathrm{C}(1)-\mathrm{C}(2)-\mathrm{N}(4)$ & $178.7(5)$ \\
$\mathrm{H}(4 \mathrm{~B}) \ldots \mathrm{N}(5)$ & 2.34 & $\mathrm{C}(2)-\mathrm{N}(3)-\mathrm{N}(5)$ & $119.6(5)$ & $\mathrm{S}(1)-\mathrm{C}(1)-\mathrm{C}(2)-\mathrm{N}(4)$ & $-0.9(6)$ \\
$\mathrm{N}(5)-\mathrm{H}(5) \ldots \mathrm{I}(1) \# 1$ & $3.989(6)$ & $\mathrm{N}(1)-\mathrm{C}(1)-\mathrm{C}(2)$ & $115.1(4)$ & & \\
$\mathrm{H}(5) \ldots \mathrm{I}(1) \# 1$ & 3.21 & $\mathrm{~N}(1)-\mathrm{C}(1)-\mathrm{S}(1)$ & $124.3(4)$ & & \\
\hline
\end{tabular}

Standard deviations in parentheses. Symmetry transformations used to generate equivalent atoms: \#1 x+1/2,-y-1/2,z+1/2 \#2-x+1/2,y-1/2,-z+1/2 \#3-x+1/2,-y-1/2,-z+1

Table 4. Selected bond lengths [in $\AA$ ], bond angles [in ${ }^{\circ}$ ] and torsion angles [in ${ }^{\circ}$ for compound $\mathbf{4 d}$

\begin{tabular}{llllll}
\hline $\mathrm{S}(1)-\mathrm{C}(1)$ & $1.750(4)$ & $\mathrm{H}(4 \mathrm{~B}) \ldots \mathrm{Br}(1) \# 1$ & 2.77 & $\mathrm{~N}(4)-\mathrm{C}(2)-\mathrm{N}(3)$ & $120.1(4)$ \\
$\mathrm{N}(4)-\mathrm{C}(2)$ & $1.297(6)$ & $\mathrm{N}(2)-\mathrm{H}(2) \ldots \mathrm{Br}(1)$ & $3.683(4)$ & $\mathrm{N}(4)-\mathrm{C}(2)-\mathrm{C}(1)$ & $121.5(4)$ \\
$\mathrm{N}(1)-\mathrm{C}(1)$ & $1.310(5)$ & $\mathrm{H}(2) \ldots \mathrm{Br}(1)$ & 2.89 & $\mathrm{~N}(3)-\mathrm{C}(2)-\mathrm{C}(1)$ & $118.3(4)$ \\
$\mathrm{N}(1)-\mathrm{N}(2)$ & $1.313(5)$ & $\mathrm{N}(3)-\mathrm{H}(3) \ldots \mathrm{Br}(1) \# 2$ & $3.343(4)$ & $\mathrm{N}(1)-\mathrm{C}(1)-\mathrm{C}(2)-\mathrm{N}(4)$ & $159.2(4)$ \\
$\mathrm{N}(3)-\mathrm{C}(2)$ & $1.327(6)$ & $\mathrm{H}(3) \ldots \mathrm{Br}(1) \# 2$ & 2.51 & $\mathrm{~S}(1)-\mathrm{C}(1)-\mathrm{C}(2)-\mathrm{N}(4)$ & $-16.6(6)$ \\
$\mathrm{N}(3)-\mathrm{N}(5)$ & $1.402(5)$ & $\mathrm{N}(5)-\mathrm{H}(5) \ldots \mathrm{Br}(1) \# 3$ & $3.470(4)$ & $\mathrm{N}(1)-\mathrm{C}(1)-\mathrm{C}(2)-\mathrm{N}(3)$ & $-17.9(6)$ \\
$\mathrm{C}(1)-\mathrm{C}(2)$ & $1.466(6)$ & $\mathrm{H}(5) \ldots \mathrm{Br}(1) \# 3$ & 2.83 & $\mathrm{~S}(1)-\mathrm{C}(1)-\mathrm{C}(2)-\mathrm{N}(3)$ & $166.2(3)$ \\
$\mathrm{N}(4)-\mathrm{H}(4 \mathrm{~A}) \ldots \mathrm{S}(1) \# 1$ & $3.353(4)$ & $\mathrm{C}(1)-\mathrm{N}(1)-\mathrm{N}(2)$ & $120.7(4)$ & $\mathrm{N}(2)-\mathrm{N}(1)-\mathrm{C}(1)-\mathrm{C}(2)$ & $-179.5(4)$ \\
$\mathrm{H}(4 \mathrm{~A}) \ldots \mathrm{S}(1) \# 1$ & 2.75 & $\mathrm{C}(2)-\mathrm{N}(3)-\mathrm{N}(5)$ & $119.7(4)$ & $\mathrm{N}(2)-\mathrm{N}(1)-\mathrm{C}(1)-\mathrm{S}(1)$ & $-4.0(6)$ \\
$\mathrm{N}(4)-\mathrm{H}(4 \mathrm{~B}) \ldots \mathrm{S}(1)$ & $3.061(4)$ & $\mathrm{N}(1)-\mathrm{C}(1)-\mathrm{C}(2)$ & $113.8(4)$ & $\mathrm{N}(5)-\mathrm{N}(3)-\mathrm{C}(2)-\mathrm{N}(4)$ & $1.1(7)$ \\
$\mathrm{H}(4 \mathrm{~B}) \ldots \mathrm{S}(1)$ & 2.64 & $\mathrm{~N}(1)-\mathrm{C}(1)-\mathrm{S}(1)$ & $126.4(3)$ & $\mathrm{N}(5)-\mathrm{N}(3)-\mathrm{C}(2)-\mathrm{C}(1)$ & $178.3(4)$ \\
$\mathrm{N}(4)-\mathrm{H}(4 \mathrm{~B}) \ldots \mathrm{Br}(1) \# 1$ & $3.454(4)$ & $\mathrm{C}(2)-\mathrm{C}(1)-\mathrm{S}(1)$ & $119.6(3)$ & & \\
\hline
\end{tabular}

Standard deviations in parentheses. Symmetry transformations used to generate equivalent atoms: \#1 -x+1/2,y+1/2,-z+1/2 \#2-x+1,-y+1,-z+1 \#3 x,y+1,z

\section{Acknowledgments}

We would like to thank the Fonds der Chemischen Industrie and the Hans-Böckler-Stiftung for financial support. 


\section{Experimental}

\section{General}

Melting points were determined with a Boëtius micro heating stage (Carl Zeiss Jena). Elemental analyses were performed with a CHNS-932 LECO analyzer. IR spectra were recorded on a Nicolet Protégé 460 FT-IR spectrometer. Mass spectra were taken on an AMD 402-3 spectrometer (Intectra $\mathrm{GmbH})$. The ${ }^{1} \mathrm{H}-\mathrm{NMR}$ and ${ }^{13} \mathrm{C}-\mathrm{NMR}$ spectra were recorded with Bruker AC 250 and ARX 300 spectrometers. The calibration of spectra was carried out by means of solvent peaks (DMSO- $\mathrm{d}_{6}: \delta^{1} \mathrm{H}=$ 2.50; $\left.\delta{ }^{13} \mathrm{C}=39.7\right)$. The assignment of signals were confirmed by DEPT and two-dimensional correlation spectra.

\section{Method A: Preparation of halides 2}

To a suspension of thiooxalic acid 2-amide 1-hydrazide 2-hydrazone (1a) $(0.67 \mathrm{~g}, 5 \mathrm{mmol})$ in methanol $(50 \mathrm{~mL})$ a solution of methyl iodide or 4-nitrobenzyl bromide in methanol $(20 \mathrm{~mL})$ was added dropwise at r.t. The mixture was stirred at r.t. for $1 \mathrm{~h}$ and then heated under reflux for $4 \mathrm{~h}$. After removing the solvent under reduced pressure a yellow oil was obtained. The addition of a small amount of ethanol gives yellow crystals which were filtered and dried. In case of $\mathbf{2} \mathbf{b}$ the unconverted 4-nitrobenzyl bromide can be removed by addition of water $(50 \mathrm{~mL})$ to the oil, filtration of the solution and evaporation of the solvent.

S-Methyl-thiooxal-1-hydrazono-2-amidrazonium iodide (2a)

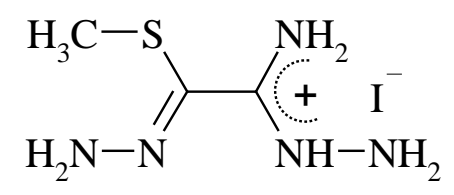

IR (KBr): $\tilde{v}\left(\mathrm{~cm}^{-1}\right)=3467,3317,3210,3125\left(\mathrm{NH}, \mathrm{NH}_{2}\right) ; 2992,2914\left(\mathrm{CH}_{3}\right) ; 1681,1605,1597,1557$, $1505\left(\mathrm{C}=\mathrm{N}, \mathrm{NH}, \mathrm{NH}_{2}\right) ; 1484(\mathrm{~S}-\mathrm{C}=\mathrm{N})$; MS (70 eV/EI): $\mathrm{m} / z(\%)=147\left(\mathrm{M}^{+}-\mathrm{HI}, 35\right) ; 128(100) ;{ }^{1} \mathrm{H}^{-}$ NMR (250.1 MHz, DMSO- $\left.d_{6}\right): \delta=10.14\left(\mathrm{~s}, 1 \mathrm{H}, \mathrm{NH}_{2} \mathrm{NH}\right) ; 9.08$ (s, 2H), 8.37 (s, 2H), 5.03 (s, 2H, $\left.\mathrm{NH}_{2}\right) ; 2.14\left(\mathrm{~s}, 3 \mathrm{H}, \mathrm{CH}_{3}\right) .{ }^{13} \mathrm{C}$ NMR $\left(62.9 \mathrm{MHz}, \mathrm{DMSO}-d_{6}\right): \delta=159.1(\mathrm{~N}-\mathrm{C}-\mathrm{N}) ; 117.2(\mathrm{~S}-\mathrm{C}=\mathrm{N}) ; 14.4$ $\left(\mathrm{CH}_{3}\right)$.

S-(4-Nitrobenzyl)thiooxal-1-hydrazono-2-amidrazonium bromide (2b)

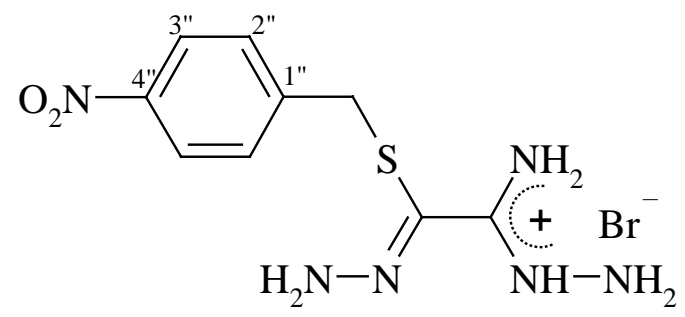


IR $(\mathrm{KBr}): \tilde{v}\left(\mathrm{~cm}^{-1}\right)=3413,3318,3296,3230\left(\mathrm{NH}, \mathrm{NH}_{2}\right) ; 3106\left(\mathrm{C}_{6} \mathrm{H}_{4}\right) ; 2935,2847\left(\mathrm{CH}_{2}\right) ; 1672$, 1602, $1564\left(\mathrm{C}=\mathrm{N}, \mathrm{NH}, \mathrm{NH}_{2}\right) ; 1519\left(\mathrm{NO}_{2}\right) ; 1504(\mathrm{~S}-\mathrm{C}=\mathrm{N}) ; 1341\left(\mathrm{NO}_{2}\right) ; \mathrm{MS}(70 \mathrm{eV} / \mathrm{EI}): \mathrm{m} / z(\%)=269$ $\left(\mathrm{M}^{+}-\mathrm{HBr}, 12\right) ; 170$ (100); ${ }^{1} \mathrm{H}-\mathrm{NMR}\left(250.1 \mathrm{MHz}, \mathrm{DMSO}-d_{6}\right): \delta=10.03$ (br, $1 \mathrm{H}, \mathrm{NH}_{2} \mathrm{NH}$ ); 9.22 (s, 2H), 8.22 (s, 2H, NH ); 7.57 (m, 2H, H-2' '); 8.13 (m, 2H, H-3' '); 4.98 (s, 2H, NH 2 ); 4.06 (s, 2H, $\mathrm{CH}_{2}$ ); ${ }^{13} \mathrm{C}-\mathrm{NMR}\left(62.9 \mathrm{MHz}, \mathrm{DMSO}-d_{6}\right): \delta=159.1(\mathrm{~N}-\mathrm{C}-\mathrm{N}) ; 146.8$ (C-4'”); 145.1 (C-1'”); $130.6(\mathrm{C}-2$ '”); $123.6\left(\mathrm{C}-3{ }^{\prime \prime}\right) ; 114.1(\mathrm{~S}-\mathrm{C}=\mathrm{N}) ; 34.5\left(\mathrm{CH}_{2}\right)$.

\section{Method B: Preparation of halides 3 and 4}

To a suspension of zwitterionic compound $\mathbf{1 b}$ or $\mathbf{1 c}(3 \mathrm{mmol})$ in methanol $(20 \mathrm{~mL})$ a solution of the corresponding alkylating agent in methanol $(50 \mathrm{~mL})$ was added dropwise at r.t. The mixture was stirred under reflux (3: $4 \mathrm{~h}, 4: 12 \mathrm{~h})$. Then the solvent was evaporated under reduced pressure. In the case of $\mathbf{3}$ addition of water to the resulting oil and leaving the mixture at $6{ }^{\circ} \mathrm{C}$ for 14 days gave yellow crystals. Compounds 4 crystallized during removing the solvent without any further manipulation. The products were purified by recrystallization ( 3 from ethanol, 4 from water). Purification of $\mathbf{4 c}$ was not necessary.

S-(4-Nitrobenzyl)thiooxal-1-(2-methylhydrazono)-2-(2-methylamidrazonium)bromide (3)

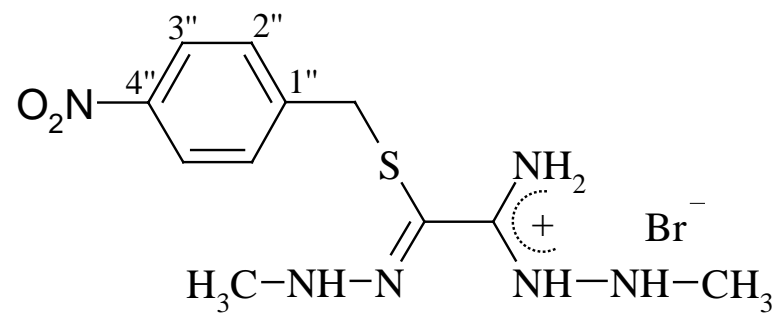

IR $(\mathrm{KBr}): \tilde{v}\left(\mathrm{~cm}^{-1}\right)=3491,3411,3368,2798\left(\mathrm{NH}, \mathrm{NH}_{2}\right) ; 1642,1597,1578(\mathrm{C}=\mathrm{N}) ; 1511\left(\mathrm{NO}_{2}\right) ; 1462$ $(\mathrm{S}-\mathrm{C}=\mathrm{N}) ; 1340\left(\mathrm{NO}_{2}\right) ; \mathrm{MS}(70 \mathrm{eV} / \mathrm{EI}): \mathrm{m} / z(\%)=296\left(\mathrm{M}^{+}-\mathrm{HBr}, 27\right) ; 28(100) ;{ }^{1} \mathrm{H}-\mathrm{NMR}(250.1 \mathrm{MHz}$, DMSO- $\left.d_{6}\right): \delta=9.84\left(\mathrm{~s}, 1 \mathrm{H}, \mathrm{CH}_{3} \mathrm{NHNH}\right) ; 9.36\left(\mathrm{q}, 1 \mathrm{H}, \mathrm{CH}_{3} \mathrm{NH}\right) ; 8.29$ (s, 1H), $8.18\left(\mathrm{~s}, 1 \mathrm{H}, \mathrm{NH}_{2}\right) ; 8.14$ (m, 2H, H-3' '); 7.53 (m, 2H, H-2' '); 5.27 (br, 1H, $\mathrm{CH}_{3} \mathrm{NH}$ ); 4.04 (s, 2H, $\mathrm{CH}_{2}$ ); 3.22 (d, 3H, $\mathrm{CH}_{3}, J=$ $3.5 \mathrm{~Hz}$ ); 2.35 (s, 3H, CH 3 ); ${ }^{13} \mathrm{C}-\mathrm{NMR}\left(62.9 \mathrm{MHz}, \mathrm{DMSO}-d_{6}\right): \delta=159.1(\mathrm{~N}-\mathrm{C}-\mathrm{N}) ; 146.8(\mathrm{C}-4$ '”); 145.1 (C-1' '); 130.8 (C-2'’); 123.6 (C-3'”); $109.1(\mathrm{~S}-\mathrm{C}=\mathrm{N}) ; 38.2\left(\mathrm{CH}_{3}\right) ; 37.3\left(\mathrm{CH}_{3}\right) ; 34.6\left(\mathrm{CH}_{2}\right)$.

S-Methyl-thiooxal-1-(2-phenylhydrazono)-2-(2-phenylamidrazonium)iodide (4a)

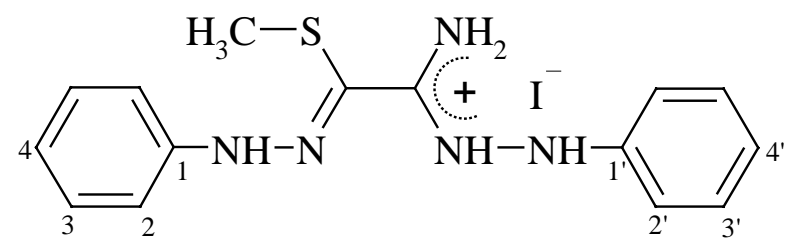


IR $(\mathrm{KBr}): \tilde{v}\left(\mathrm{~cm}^{-1}\right)=3384,3285\left(\mathrm{NH}_{2}\right) ; 3171(\mathrm{NH}) ; 1645(\mathrm{C}=\mathrm{N}) ; 1515 ; 1469 ; 1240 ; 758 ;$ MS (70 $\mathrm{eV} / \mathrm{EI}): m / z(\%)=299\left(\mathrm{M}^{+}-\mathrm{HI}, 100\right) ;{ }^{1} \mathrm{H}-\mathrm{NMR}\left(300.1 \mathrm{MHz}\right.$, DMSO- $\left.d_{6}\right): \delta=11.12(\mathrm{~s}, 1 \mathrm{H}$, $\left.\mathrm{C}_{6} \mathrm{H}_{5} \mathrm{NHNH}\right) ; 11.02\left(\mathrm{~s}, 1 \mathrm{H}, \mathrm{C}_{6} \mathrm{H}_{5} \mathrm{NHN} \underline{\mathrm{H}}\right) ; 9.31$ (s, $\left.1 \mathrm{H}\right), 9.20\left(\mathrm{~s}, 1 \mathrm{H}, \mathrm{NH}_{2}\right) ; 8.47$ (s, 1H, $\left.\mathrm{C}_{6} \mathrm{H}_{5} \mathrm{NHN}\right)$; 7.72 (m, 2H, H-2'); 7.36 (m, 2H, H-3'); 7.29 (m, 2H, H-3); 7.06 (m, 1H, H-4'); 6.91 (m, 1H, H-4); $6.88(\mathrm{~m}, 1 \mathrm{H}, \mathrm{H}-2) ; 2.36\left(\mathrm{~s}, 3 \mathrm{H}, \mathrm{CH}_{3}\right) ;{ }^{13} \mathrm{C}-\mathrm{NMR}\left(75.5 \mathrm{MHz}, \mathrm{DMSO}-d_{6}\right): \delta=160.6(\mathrm{~N}-\mathrm{C}-\mathrm{N}) ; 146.8$ (C-1); 142.5 (C-1'); 129.3, 129.2 (C-3, C-3'); 123.6 (C-4'); 120.9 (C-4); 119.5 (S-C=N); 116.1 (C-2'); 113.7 (C-2); $15.9\left(\mathrm{CH}_{3}\right)$.

S-Ethyl-thiooxal-1-(2-phenylhydrazono)-2-(2-phenylamidrazonium)iodide (4b)

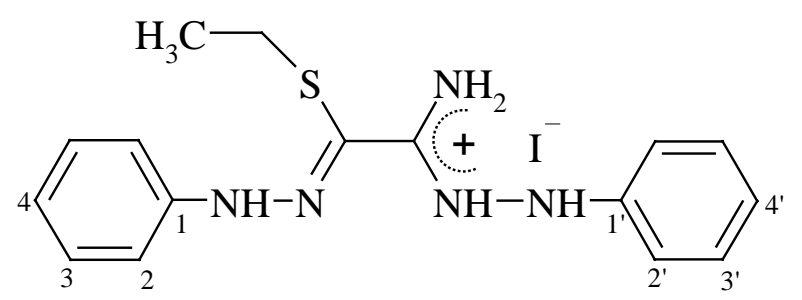

IR $(\mathrm{KBr}): \tilde{v}\left(\mathrm{~cm}^{-1}\right)=3389,3286\left(\mathrm{NH}_{2}\right) ; 3176(\mathrm{NH}) ; 1645(\mathrm{C}=\mathrm{N}) ; 1518 ; 1468 ; 1235 ; 760 ;$ MS $(70$ $\mathrm{eV} / \mathrm{EI}): \mathrm{m} / \mathrm{z}(\%)=313\left(\mathrm{M}^{+}-\mathrm{HI}, 100\right) ;{ }^{1} \mathrm{H}-\mathrm{NMR}\left(250.1 \mathrm{MHz}\right.$, DMSO- $\left.d_{6}\right): \delta=11.15(\mathrm{~s}, 1 \mathrm{H}$,

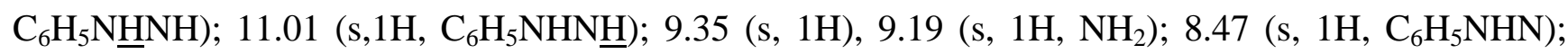
7.73 (m, 2H, H-2'); 7.36 (m, 2H, H-3'); 7.29 (m, 2H, H-3); 7.07 (m, 1H, H-4'); 6.91 (m, 1H, H-4); $6.84(\mathrm{~m}, 2 \mathrm{H}, \mathrm{H}-2) ; 2.88$ (q, 2H, $\left.\mathrm{CH}_{2}, J=7.3 \mathrm{~Hz}\right) ; 1.24\left(\mathrm{t}, 3 \mathrm{H}, \mathrm{CH}_{3}, J=7.3 \mathrm{~Hz}\right) ;{ }^{13} \mathrm{C}-\mathrm{NMR}(62.9 \mathrm{MHz}$, DMSO- $\left.d_{6}\right): \delta=161.1(\mathrm{~N}-\mathrm{C}-\mathrm{N}) ; 146.8$ (C-1); 142.4 (C-1'); 129.3, 129.2 (C-3, C-3'); 123.7 (C-4'); $120.9(\mathrm{C}-4) ; 117.7(\mathrm{~S}-\mathrm{C}=\mathrm{N}) ; 116.1\left(\mathrm{C}-2^{\prime}\right) ; 113.6(\mathrm{C}-2) ; 27.4\left(\mathrm{CH}_{2}\right) ; 14.8\left(\mathrm{CH}_{3}\right)$.

S-Decyl-thiooxal-1-(2-phenylhydrazono)-2-(2-phenylamidrazonium)bromide (4c)

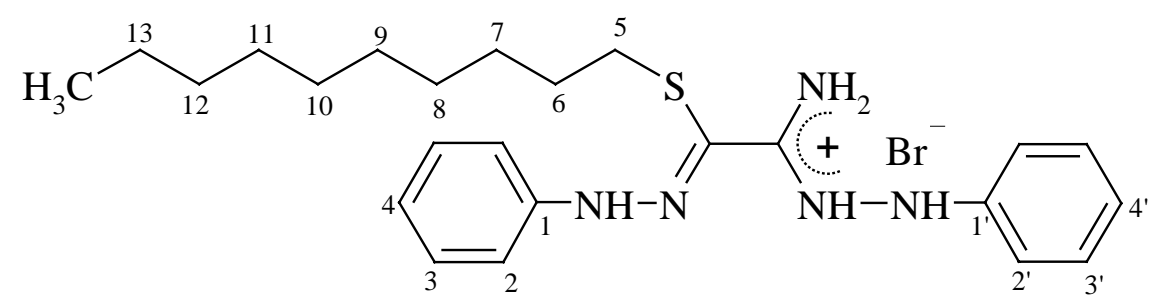

IR $(\mathrm{KBr}): \tilde{v}\left(\mathrm{~cm}^{-1}\right)=3054,2955\left(\mathrm{NH}_{2}\right) ; 2924(\mathrm{NH}) ; 1646,1601(\mathrm{C}=\mathrm{N}) ; 1497 ; 1464 ; 1240 ; 753$; MS $(70 \mathrm{eV} / \mathrm{EI}): m / z(\%)=426\left(\mathrm{M}^{+}-\mathrm{HBr}, 100\right) ;{ }^{1} \mathrm{H}-\mathrm{NMR}\left(250.1 \mathrm{MHz}, \mathrm{DMSO}-d_{6}\right): \delta=11.10(\mathrm{~s}, 1 \mathrm{H}$, $\left.\mathrm{C}_{6} \mathrm{H}_{5} \mathrm{~N} \underline{\mathrm{HNH}}\right) ; 11.01\left(\mathrm{~s}, 1 \mathrm{H}, \mathrm{C}_{6} \mathrm{H}_{5} \mathrm{NHN} \underline{\mathrm{H}}\right) ; 9.36(\mathrm{~s}, 1 \mathrm{H}), 9.20\left(\mathrm{~s}, 1 \mathrm{H}, \mathrm{NH}_{2}\right) ; 8.51$ (s, $\left.1 \mathrm{H}, \mathrm{C}_{6} \mathrm{H}_{5} \mathrm{NHN}\right)$; 7.72 (m, 2H, H-2'); 7.35 (m, 2H, H-3'); 7.28 (m, 2H, H-3); 7.05 (m, 1H, H-4'); 6.91 (m, 1H, H-4); 6.84 (m, 2H, H-2); 2.85 (t, 2H, $\mathrm{SCH}_{2}, J=7.3 \mathrm{~Hz}$ ); 1.65-1.15 (m, 16H, H-6-H-13); 0.83 (t, 3H, $\mathrm{CH}_{3}, J$ $=7.3 \mathrm{~Hz}) ;{ }^{13} \mathrm{C}-\mathrm{NMR}\left(62.9 \mathrm{MHz}, \mathrm{DMSO}-d_{6}\right): \delta=161.0(\mathrm{~N}-\mathrm{C}-\mathrm{N}) ; 146.8(\mathrm{C}-1) ; 142.5\left(\mathrm{C}-1^{\prime}\right) ; 129.3$, 129.2 (C-3, C-3'); 123.6 (C-4'); 120.8 (C-4); 118.1 (S-C=N); 116.0 (C-2'); 113.6 (C-2); $33.0\left(\mathrm{SCH}_{2}\right)$; 31.5 (C-12); 29.6, 29.1 (2x), 28.9 (2x), 28.3 (C-6 - C-11); $22.3(\mathrm{C}-13) ; 14.2\left(\mathrm{CH}_{3}\right)$. 
S-Benzyl-thiooxal-1-(2-phenylhydrazono)-2-(2-phenylamidrazonium)bromide (4d)

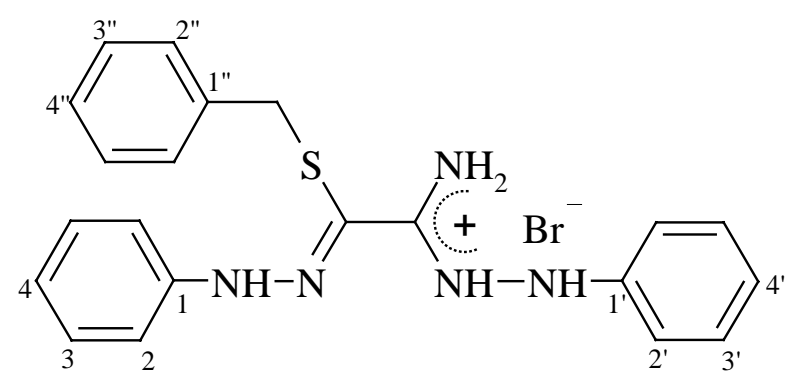

IR $(\mathrm{KBr}): \tilde{v}\left(\mathrm{~cm}^{-1}\right)=3380,3279,3181\left(\mathrm{NH}_{2}\right) ; 3053(\mathrm{NH}) ; 1644,1597(\mathrm{C}=\mathrm{N}) ; 1519 ; 1497 ; 1469$; 1230; 756; MS (70 eV/EI): $m / z(\%)=375\left(\mathrm{M}^{+}-\mathrm{HBr}, 45\right) ; 91$ (100); ${ }^{1} \mathrm{H}-\mathrm{NMR}\left(250.1 \mathrm{MHz}, \mathrm{DMSO}-d_{6}\right)$ : $\delta=11.19\left(\mathrm{~s}, 1 \mathrm{H}, \mathrm{C}_{6} \mathrm{H}_{5} \mathrm{~N} \underline{\mathrm{HNH}}\right) ; 10.82\left(\mathrm{~s}, 1 \mathrm{H}, \mathrm{C}_{6} \mathrm{H}_{5} \mathrm{NHN} \underline{\mathrm{H}}\right) ; 9.20(\mathrm{~s}, 1 \mathrm{H}), 8.91\left(\mathrm{~s}, 1 \mathrm{H}, \mathrm{NH}_{2}\right) ; 8.42(\mathrm{~s}, 1 \mathrm{H}$, $\mathrm{C}_{6} \mathrm{H}_{5} \mathrm{NHN}$ ); 7.72 (m, 2H, H-2'); 7.45-7.20 (m, 9H, H-2', H-3, H-3', H-3', H-4' '); 7.07 (m, 1H, H$\left.4^{\prime}\right) ; 6.89$ (m, 1H, H-4); 6.63 (m, 2H, H-2); 4.15 (s, 2H, $\left.\mathrm{CH}_{2}\right) ;{ }^{13} \mathrm{C}-\mathrm{NMR}\left(62.9 \mathrm{MHz}, \mathrm{DMSO}-d_{6}\right): \delta=$ 160.9 (N-C-N); 146.7 (C-1); 142.3 (C-1'); 136.7 (C-1'”); 129.3, 129.2 (2x), 128.6 (C-2', C-3, C-3', C3'’); 127.8 (C-4'’); 123.8 (C-4'); 120.7 (C-4); 116.7 (S-C=N); 116.3 (C-2'); 113.6 (C-2); $36.2\left(\mathrm{CH}_{2}\right)$.

S-(4-Nitrobenzyl)thiooxal-1-(2-phenylhydrazono)-2-(2-phenylamidrazonium)bromide (4e)

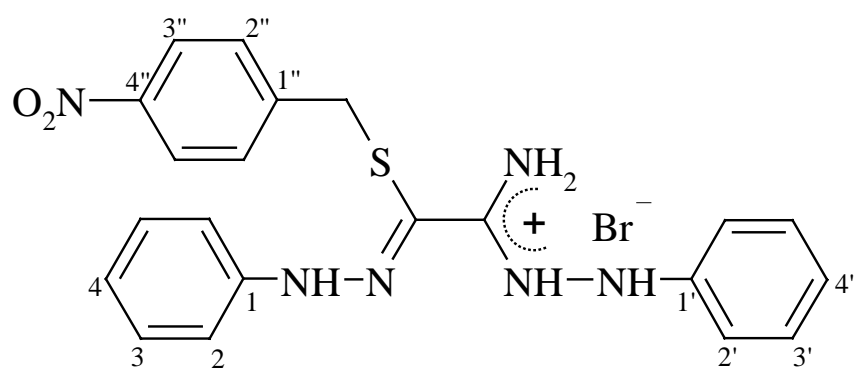

IR $(\mathrm{KBr}): \tilde{v}\left(\mathrm{~cm}^{-1}\right)=3352(\mathrm{NH}) ; 3262,3165\left(\mathrm{NH}_{2}\right) ; 3079(\mathrm{NH}) ; 1604(\mathrm{C}=\mathrm{N}) ; 1525\left(\mathrm{NO}_{2}\right) ; 1496$; 1475; $1346\left(\mathrm{NO}_{2}\right) ; 1256 ; 753$; MS (70 eV/EI): $\mathrm{m} / z(\%)=420\left(\mathrm{M}^{+}-\mathrm{HBr}, 100\right) ;{ }^{1} \mathrm{H}-\mathrm{NMR}(250.1 \mathrm{MHz}$, DMSO- $\left.d_{6}\right): \delta=11.25$ (s,1H, $\left.\mathrm{C}_{6} \mathrm{H}_{5} \mathrm{NHNH}\right) ; 10.82\left(\mathrm{~s}, 1 \mathrm{H}, \mathrm{C}_{6} \mathrm{H}_{5} \mathrm{NHNH}\right) ; 9.19$ (s, $\left.1 \mathrm{H}\right), 8.96\left(\mathrm{~s}, 1 \mathrm{H}, \mathrm{NH}_{2}\right)$; 8.40 (s, 1H, $\left.\mathrm{C}_{6} \mathrm{H}_{5} \mathrm{NHN}\right) ; 8.17$ (m, 2H, H-3' '); 7.71 (m, 2H, H-2'); 7.62 (m, 2H, H-2'’); 7.34 (m, 2H, H3'); 7.21 (m, 2H, H-3); 7.08 (m, 1H, H-4'); 6.88 (m, 1H, H-4); 6.62 (m, 2H, H-2); 4.26 (s, 2H, $\mathrm{CH}_{2}$ ); ${ }^{13} \mathrm{C}-\mathrm{NMR}\left(62.9 \mathrm{MHz}, \mathrm{DMSO}-d_{6}\right): \delta=161.0(\mathrm{~N}-\mathrm{C}-\mathrm{N}) ; 146.8,146.7$ (C-1, C-1' '); 145.2 (C-4'”); 142.2 (C-1'); 130.8 (C-2'’); 129.1 (2x) (C-3, C-3'); 124.0 (C-4'); 123.7 (C-3'’); 120.8 (C-4); 116.4 (C-2'); $115.8(\mathrm{~S}-\mathrm{C}=\mathrm{N}) ; 113.5(\mathrm{C}-2) ; 35.4\left(\mathrm{CH}_{2}\right)$.

\section{X-Ray structure determinations}

Crystals of $\mathbf{4 b}$ and $\mathbf{4 d}$ were sealed onto glass fibres and mounted on a Bruker P4 automated four circle diffractometer (Mo- $\mathrm{K}_{\alpha}$ radiation, $\lambda=0.71073 \AA$, graphite monochromator). Prior to the automatic cell determination and data collection routines rotational photos were taken to check the 
quality of the crystals. The data collection was done in routine $\omega$-scan. Afterwards a Psi-scan was performed in order to do calculations with respect to absorption correction. The structures were solved by direct methods (Bruker SHELXTL) and refined by the full-matrix least-squares method of SHELXL-97 (G. M. Sheldrick, Universität Göttingen, 1997). Non-hydrogen atoms were refined with anisotropic displacement parameters. Important crystal data as well as bond parameters can be taken from Tables 1-4. Due to the presence of hydrogen donating and accepting groups attempts were made to elucidate the positions of the hydrogen atoms within the NH-groups from the difference maps. Except the hydrogen at N5 in $\mathbf{4 b}$ all other positions could be located. These hydrogen position were refined freely while the rest was refined according to the riding model. Crystallographic data (excluding structure factors) reported in this paper have been deposited with the Cambridge Crystallographic Data Centre as Supplementary Publication No. CCDC-155603 (4b) and CCDC155604 (4d). Copies of the data can be obtained free of charge on application to The Director, CCDC, 12 Union Road, Cambridge CB2 1EZ, UK (fax: +44 (1223)336033; e-mail: deposit@ccdc.cam.ac.uk ).

\section{References and Notes}

1. Bauer, W.; Kühlein, K. In Methoden der organischen Chemie (Houben-Weyl): Carbonsäuren und Carbonsäure-Derivate; Falbe, J., Ed.; Georg Thieme Verlag: Stuttgart, New York, 1985, Vol. E5, Part 2, pp 1263, 1300.

2. Jensen, K. A.; Pedersen, C. Studies of Thioacids and Their Derivatives. VI. Formation of Thiadiazoles and Tetrazines in the Preparation of Thiohydrazides. Acta Chem. Scand. A 1961, 15, 1124-1129.

3. Dehne, H.; Scheunemann, A.; Michalik, M.; Hartung, H.; Heinemann, F.; Kibbel, H. U. Thiooxalsäure-2-amid-1-hydrazid-2-hydrazon: Eine neue zwitterionische Verbindung. Phosphorus, Sulfur Silicon Relat.Elem. 1994, 86, 177-179.

4. Drexler, K.; Dehne, H.; Reinke, H.; Michalik, M. Reactions of Sodium Cyanodithioformate with Monosubstituted Hydrazines. Liebigs Ann./Recueil 1997, 269-271.

5. Drexler, K.; Dehne, H.; Reinke, H.; Michalik, M. Reactions of Zwitterionic Thiooxamic Acid Derivatives with Alkyl Bromoacetates. Sulfur Lett. 1998, 21, 163-177.

6. Dehne, H.; Drexler, K.; Martens, K.; Reinke, H.; Michalik, M. Zwitterionic Thiooxamic Acid Derivatives as Efficient Building Blocks for Biheterocycles. Sulfur Lett. 2000, 24, 29-37.

Sample Availability: Samples of compounds $\mathbf{1 a - c}, \mathbf{2 a}, \mathbf{2 b}$ and $\mathbf{4 b}$ are available from MDPI.

(C) 2001 by MDPI (http://www.mdpi.org). Reproduction is permitted for noncommercial purposes 